\title{
Cambio climático y modelado de distribución de especies de Stevia (Asteraceae) en el noroeste de la Argentina
}

\author{
Juan F. Rodríguez-Cravero ${ }^{1, \bigotimes ;}$; Mariana A. Grossi²; Taryn Fuentes-Castillo ${ }^{3}$ \& \\ Diego G. Gutiérrez'
}

${ }^{1}$ División Plantas Vasculares, Museo Argentino de Ciencias Naturales (MACN-CONICET). Buenos Aires, Argentina. ${ }^{2}$ División Plantas Vasculares, Museo de La Plata (FCNyM, UNLP). La Plata, Argentina. ${ }^{3}$ Departamento de Ecología, Facultad de Ciencias Biológicas, Pontificia Universidad Católica de Chile. Santiago, Chile.

\begin{abstract}
RESUmen. La elevada biodiversidad y riqueza específica, junto con una gran variedad de ecosistemas, convierten al noroeste de la Argentina (NOA) en un área adecuada para estudiar los posibles efectos del cambio climático sobre la distribución de los organismos. Asteraceae es una familia de plantas con flores bien representada en esta área; en especial, el género Stevia, con 25 especies que habitan diferentes ambientes. Los objetivos de este estudio fueron: 1) modelar la aptitud de hábitat para cinco especies de Stevia que habitan el noroeste argentino, y 2) proyectar dicha aptitud bajo un posible escenario de emisión de gases de efecto invernadero para el año 2050. Se analizaron las especies S. alpina, S. breviaristata, S. minor, S. vaga y S. yaconensis. Los resultados mostraron que, al comparar los mapas basados en condiciones climáticas actuales y futuras, todas las especies de Stevia sufrirían modificaciones en sus rangos de aptitud. Las tendencias observadas en ambos escenarios climáticos futuros son similares, aunque más drásticas en el modelo rcp8.5. El área de aptitud de Stevia alpina, S. breviaristata y S. minor se vería reducida, mientras que la de $S$. vaga y $S$. yaconensis aumentaría. Excepto $S$. minor, las especies presentaron una tendencia principal a migrar del este al oeste, un fenómeno conocido como desplazamiento en altura. Stevia minor sería la especie más afectada en un escenario de mayor emisión de gases; sufriría una reducción mayor a $80 \%$ para valores de aptitud superiores a 0.65 .
\end{abstract}

[Palabras clave: distribución potencial, nicho climático]

\begin{abstract}
Aвstract. Climatic change and species modeling of Stevia (Asteraceae) in northwestern Argentina. The high biodiversity and species richness, along with a great variety of ecosystems, turn northwestern Argentina into an adequate area for study the possible effects of climate change on the distribution of organisms. Asteraceae is a well-represented family of flowering plants in this area; especially, the genus Stevia, with 25 species which inhabit different environments. The goals of this study were: 1) to model habitat suitability for five species of Stevia distributed in northwestern Argentina, and 2) to project such suitability under a possible greenhouse gas emission scenario by 2050. Species S. alpina, S. breviaristata, S. minor, S. vaga and S. yaconensis were analyzed. Results showed that, when comparing maps based on current and future climatic conditions, all Stevia species would undergo changes in their fitness ranges. The trends observed in both future climate scenarios are similar, although they show more drastic consequences in the rcp 8.5 model. Stevia alpina, S. breviaristata and S. minor reduce their area of aptitude, while $S$. vaga and $S$. yaconensis experience an increase of said area. Except for $S$. minor, the species showed a major tendency to migrate from East to West, a phenomenon known as altitude shift. Stevia minor would be the more affected species in a higher gas emission scenario since it will suffer a reduction greater than $80 \%$ for its suitability values greater than 0.65 .
\end{abstract}

[Keywords: climatic niche, potential distribution]

\section{INTRODUCCIÓN}

Actualmente, la biósfera experimenta un cambio climático acelerado que amenaza la biodiversidad a nivel global debido a un aumento continuo en la tasa de extinción de especies (Pimm et al. 1995; Higgins 2007). Este fenómeno constituiría la causa principal de la reducción y expansión de las distribuciones de diversas especies, ya que altera al mismo tiempo la composición y la dinámica de las comunidades (Parmesan 2006; Moritz et al. 2008). El Panel Intergubernamental por el Cambio Climático (IPCC, siglas en inglés) estima un aumento global de la temperatura

Editora asociada: Adriana Ruggiero

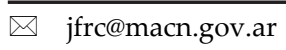

en $0.2{ }^{\circ} \mathrm{C}$ por cada década futura (IPCC 2007), lo que podría alterar los patrones conocidos de la biota en la Tierra. No todos los ambientes terrestres son afectados de la misma forma; los montañosos son los que muestran los indicios más claros de amplitud en las variaciones de temperatura y, a su vez, son los más afectados por la proporción elevada de especies endémicas o de nicho climático restringido que conforman estos ecosistemas (Beniston et al. 1997; Cuesta-Camacho et al. 2008). Por lo tanto, desde el punto de vista de la seguridad ambiental, la hidrología, la biodiversidad, la regulación climática y la fuente de genes, las

Recibido: 9 de mayo de 2016

Aceptado: 1 de septiembre de 2017 
montañas tienen una importancia "per se" que justificarían una protección y conservación cuidadosa (González 2005).

El noroeste argentino (NOA) presenta una heterogeneidad climática asociada principalmente a la presencia de masas húmedas a lo largo de diferentes límites altitudinales (Minneti 2005). Asimismo, la topografía de la región genera variaciones climáticas que van desde climas cálidos y húmedos (subtropical) en la parte norteoriental de la región, cálidos y secos (áridos) en la parte central, y frío y seco (árido) en la parte sur-occidental (Minneti 2005).

El NOA incluye las ecorregiones de Altos Andes, Chaco Seco, Monte de Sierras y Bolsones, Puna y Yungas (Brown and Pacheco 2006). Mendoza y González (2011) dividen algunas de estas unidades según la clasificación climática de Köppen (Köppen 1923). De esta forma, el Chaco serrano ("sensu" Brown and Pacheco 2006) queda redefinido por el Chaco árido (con un tipo de clima árido de desierto [caliente]), Chaco semiárido (semiárido de estepa [caliente]) y Chaco serrano (templado frío de invierno seco [con verano caluroso o fresco] y semiárido de estepa [caliente o frío]). La ecorregión de la Puna se divide en Puna (clima seco de alta montaña) y Prepuna (semiárido de estepa [muy frío]). La ecorregión Montes de Sierras y Bolsones se descompone en Prepuna y Monte (ambas con clima de tipo árido de desierto [caliente, frío y muy frío]). La ecorregión de las Yungas queda definida por Yungas sensu stricto (clima templado de invierno seco [que puede ser frío con verano caluroso o fresco, o muy frío con verano fresco]). La región de los Altos Andes se define como Altoandina y posee un clima seco de alta montaña. Bajo estas consideraciones, los límites de las ecorregiones son semejantes desde un marco climático y florístico (Mendoza and González 2011).

Al presente, se han documentado cambios significativos en las temperaturas, aumento paulatino en regímenes de precipitación y cambios en los patrones climáticos estacionales de la región, y una tendencia a la aridización en las zonas del Altiplano (e.g., Minetti and González 2006; Gonzáles 2009; Marengo et al. 2011; Carrilla et al. 2013; Morales et al. 2015), y también modificaciones en las condiciones edáficas producto de actividades antrópicas (e.g., Grau et al. 2005). En relación al cambio climático proyectado, a escala del Altiplano se espera una disminución significativa de las precipitaciones al final de este siglo (Minvielle and Garreaud 2011) y un aumento de las temperaturas, especialmente a mayor altitud (Urrutia and Vuille 2009). A pesar de esto, son pocos los estudios que han evaluado el efecto que el cambio climático podría tener sobre los organismos que allí habitan, en particular sobre las plantas (Godoy-Bürki 2016). En el NOA se identificaron varias áreas de endemismo (Aagesen et al. 2012; Szumik et al. 2012; Godoy-Bürki 2015; Godoy- Bürki et al. 2017). Por otro lado, la alta diversidad y riqueza específica, sumado a la variedad de altitudes que presentan los Andes tropicales, convierten al NOA en una zona potencialmente vulnerable al cambio climático y sus consecuencias (Bush 2002; Malcolm et al. 2006; Vuille et al. 2008; Grossi et al. 2012; Godoy-Bürki 2016; Godoy- Bürki et al. 2017).

El NOA posee una gran diversidad de especies de Asteraceae (Katinas et al. 2007; Grossi et al. 2012) y, en particular, de Stevia Cav. En esta región habitan 25 de las 33 especies que se distribuyen en la Argentina (Freire 2008; Freire and Ariza 2014). La mayoría de estas especies (22) son endémicas del país. Stevia es uno de los géneros más diversos de la tribu Eupatorieae. Presenta 235 especies y se distribuye en el continente americano desde el sur de los Estados Unidos hasta el desierto costero en Chile y el norte de la Patagonia argentina (Cabrera and Freire 1997; Hind and Robinson 2007; Freire 2008; Freire and Ariza 2014; Gutiérrez et al. 2016).

El modelado de distribución de especies (SDM, su sigla en inglés) se puede utilizar para generar hipótesis de cambios geográficos en las condiciones favorables donde las especies tienen lugar, mediante el uso de datos de presencia de los taxones en localidades puntuales, en conjunto con datos climáticos (Peterson et al. 2002; Thomas et al. 2004; Guisan and Thuiller 2005; Ferrier and Guisan 2006; Pliscoff and FuentesCastillo 2011). Estos modelos trabajan sobre la idea del nicho potencial o fundamental de las especies (Hutchinson 1957) y pueden contribuir a entender la adaptabilidad y las preferencias climáticas de las especies vegetales (Scheldeman et al. 2006), a generar estrategias para la conservación (Coitiño et al. 2013), a llenar los vacíos de colección (Jarvis et al. 2005) y a evaluar el daño potencial de las especies invasoras (Biganzoli et al. 2013; Heit et al. 2013; Young et al. 2013; Vilatersana et al. 2016). 
El presente estudio tiene como objetivo indagar sobre las variables climáticas (o ambientales) que podrían influir en la distribución actual, y bajo un escenario de cambio climático, de cinco especies del género Stevia, en el noroeste argentino. Este trabajo representa el punto de partida en el estudio sobre la distribución y ecología de las especies de Stevia de la Argentina.

\section{Materiales y Métodos}

\section{Área de estudio}

El área de estudio incluyó las provincias de Catamarca, Jujuy, La Rioja, Salta y Tucumán, correspondientes a la región noroeste de Argentina (latitud: $-69.75^{\circ},-62.35^{\circ}$; longitud: $-32^{\circ},-21.75^{\circ}$ ), denominada NOA (Figura 1). $\mathrm{El}$ área seleccionada presenta una superficie aproximada de $423500 \mathrm{~km}^{2}$. Los límites de las unidades ecorregionales siguen a Brown y Pacheco (2006) por su fácil comprensión, y los tipos de clima de la región, a Mendoza y González (2011). Las ecorregiones que se encuentran representadas en el área de estudio son: Altos Andes (AA), Chaco Seco (CH), Monte de Sierras y Bolsones (MO), Puna (PU), Selva de Yungas (YU) (Brown and Pacheco 2006). El gradiente altitudinal varía desde los 500 m s. n. m. en el este hasta valores superiores a $4000 \mathrm{~m}$ s.n.m. hacia el oeste (Cabrera 1976). El gradiente de precipitación oscila entre 900-3500 mm anuales en la cara oriental de los Andes y menos de 100 mm/ año en la vertiente occidental (Godoy-Bürki 2016).

\section{Especies seleccionadas del género Stevia}

De las 25 especies de Stevia que crecen en el área de estudio (Freire 2008; Freire and Ariza Espinar 2014), cinco fueron seleccionadas para el análisis: S. alpina Griseb. (Figura 2A), S. breviaristata Hook. and Arn., S. minor Griseb. (Figura 2B), S. vaga Griseb. y S. yaconensis Hieron. (Figura 2C). Estas especies de Stevia se seleccionaron por ser las que están mejor representadas en el área de estudio, porque existen trabajos para la correcta identificación taxonómica de las especies de este género en la Argentina, porque ocupan diferentes

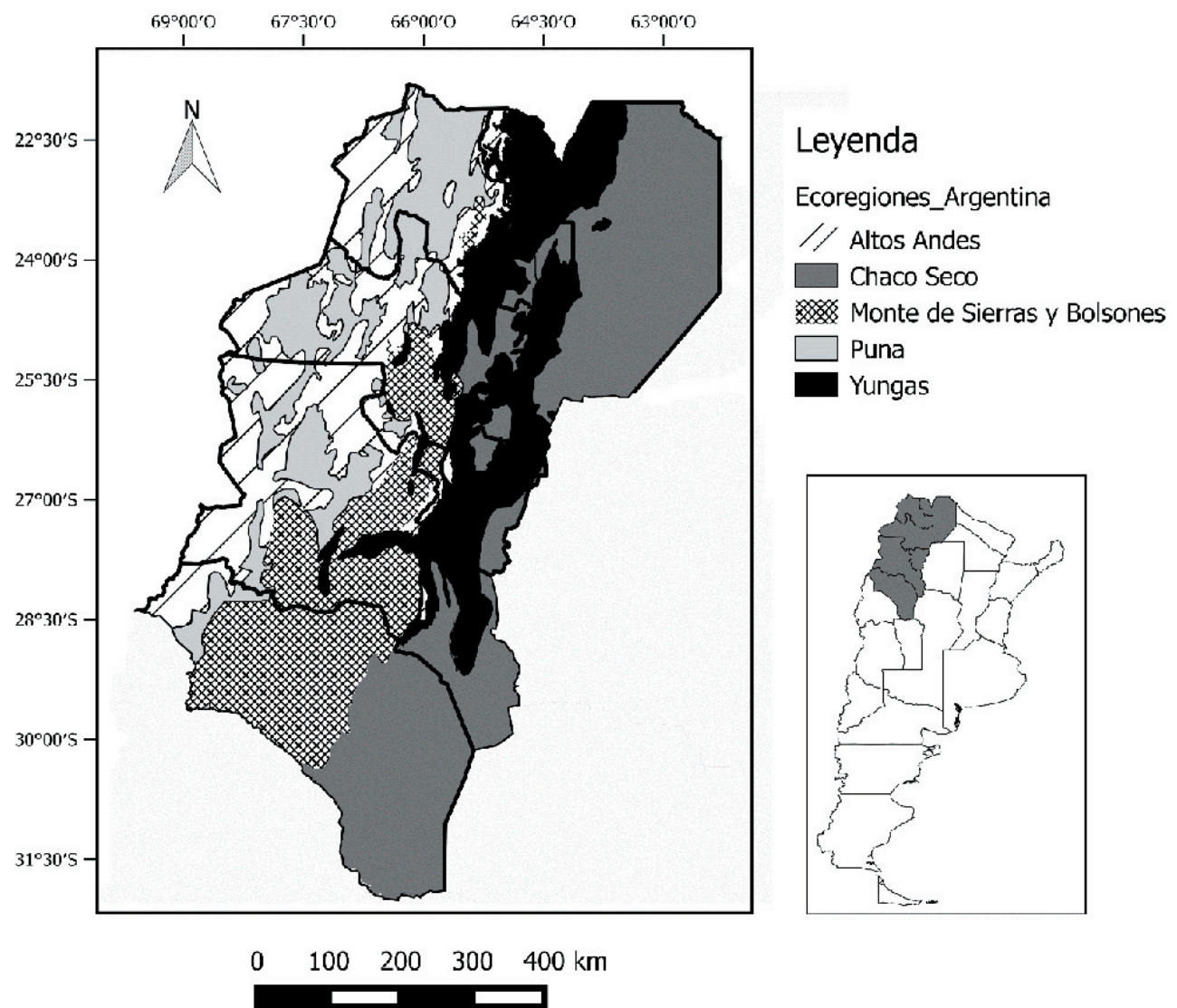

Figura 1. Mapa del noroeste argentino (NOA) y sus ecorregiones (según Brown and Pacheco [2006]).

Figure 1. Map of the northwestern Argentina (NOA) and its ecoregions (sensu Brown and Pacheco [2006]). 

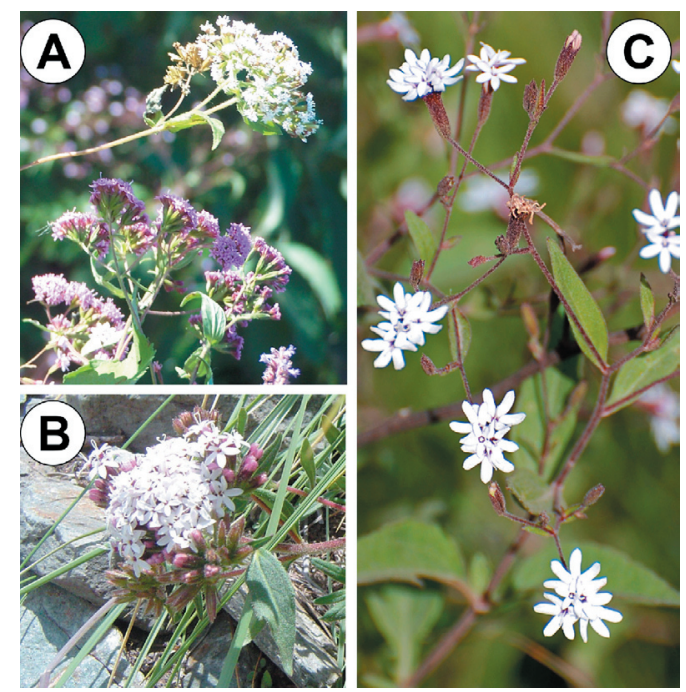

Figura 2. Especies de Stevia del Noroeste Argentino. A) Stevia alpina, B) Stevia minor, C) Stevia yaconensis. Fotos: Lázaro Novara.

Figure 2. Species of Stevia from the Argentine Northwest. A) Stevia alpina, B) Stevia minor, C) Stevia yaconensis. Photos: Lázaro Novara.

ambientes y ecorregiones, y por la presencia de más de 15 ocurrencias geográficas no correlacionadas dentro del área de estudio (ver Apéndice 1). De las especies seleccionadas, $S$. alpina se caracteriza por ser endémica del área de estudio. El resto de las especies son nativas y características del área, con una distribución un poco más amplia (Tabla 1). En el caso particular de S. breviaristata, S. minor y S. yaconensis, el rango de distribución se extendería hasta Bolivia (Beck et al. 2016). Dado que hasta el momento no existen trabajos sistemáticos o florísticos que permitan una identificación correcta de las especies de Stevia en Bolivia, los especímenes de ese país no fueron incluidos en el presente estudio. Por otro lado, en el caso de aquellas especies que presentan variedades taxonómicas (Tabla 1), dichas categorías infraespecíficas se consideraron parte del conjunto del taxón respectivo. De las especies seleccionadas, $S$. alpina, S. breviaristata, S. vaga y S. yaconensis fueron relevadas en zonas transformadas por la agricultura y que actualmente representan una zona de transición climática (Mendoza and González 2011).

Stevia alpina habita en zonas con clima templado moderado, lluvioso y con invierno seco no riguroso (templado de pradera, un clima asociado con las Selvas Montanas y Pedemontanas de las Yungas), así como en regiones de clima semiárido de estepa (Chaco Serrano) a árido de desierto (Monte de Sierras y Bolsones). Las precipitaciones varían desde
$500 \mathrm{~mm} /$ año en las zonas más secas a $2500 \mathrm{~mm} /$ año en las Selvas Montanas. Stevia breviaristata está presente mayormente en las Yungas, en climas templados a semiáridos, calientes (temperatura $>22^{\circ} \mathrm{C}$ ) o fríos (temperatura $<18$ $\left.{ }^{\circ} \mathrm{C}\right)$. En el Chaco Serrano se distribuye por climas semiáridos calientes. No fue relevada en zonas con precipitaciones menores a 450 $\mathrm{mm} / \mathrm{año}$. A Stevia minor se la considera parte de la vegetación de altura, característica de climas secos que van de fríos a muy fríos (temperatura $<10^{\circ} \mathrm{C}$ ). Corresponde con climas semiáridos muy fríos, áridos de desiertos fríos y muy fríos, y secos de alta montaña, también denominados polares de altura de planicies continentales (precipitaciones anuales menores a $250 \mathrm{~mm}$ ). Stevia vaga fue relevada en las ecorregiones de Monte de Sierras y Bolsones y Yungas, en zonas de climas secos que van de templados a zonas de transición con climas áridos, y de temperaturas frías a muy frías. Por último, S. yaconensis se distribuye tanto en Yungas y Chaco Serrano como en zonas más elevadas de Monte de Sierras y Bolsones. Habita en regiones templadas de verano fresco, semiáridas calientes y en zonas áridas muy frías al norte de su distribución.

Se analizaron especímenes de los siguientes herbarios: $\mathrm{BA}, \mathrm{BAB}, \mathrm{BAF}, \mathrm{CORD}, \mathrm{CTES}$, LIL, LP y SI (Thiers 2013). Las localidades utilizadas para el modelado fueron tomadas de las etiquetas de los ejemplares; previamente se había verificado su correcta determinación taxonómica. La asignación precisa de la identidad taxonómica de los ejemplares analizados resulta crucial para evaluar correctamente la distribución potencial de las especies, pues se trata del dato de origen de los análisis. Incorporar registros erróneos al modelado afecta a los patrones espaciales resultantes y puede determinar la sobreestimación de las áreas de distribución (Maldonado et al. 2015). Por ello no se relevaron ejemplares de bases de datos que no pudieran ser verificados taxonómicamente. Con los datos de presencia en el área de estudio de todos los especímenes de las cinco especies seleccionadas se procedió a georreferenciar 124 localidades (ver Apéndice 1 en Materiales Suplementarios) por medio del programa Google Earth y el protocolo establecido por Spencer et al. (2006). Aunque las especies $S$. breviaristata, S. minor y S. yaconensis extenderían su distribución a Bolivia, no existen trabajos sistemáticos del género que garanticen la correcta posición taxonómica de las especies en la región. No se consideraron en este análisis los materiales observados de Bolivia; en esta 
Tabla 1. Especies de Stevia estudiadas. Provincias político-administrativas (Argentina). CAT: Catamarca; JUJ: Jujuy; SAL: Salta; LRI: La Rioja; TUC: Tucumán; departamentos (Bolivia). CH: Chuquisaca; CO: Coquimbo; LP: La Paz; OR: Oruro; PO: Potosí; SC: Santa Cruz; TA: Tarija. Ecorregiones. AA: Altos Andes; CS: Chaco Seco; MO: Monte de Sierras y Bolsones; PU: Puna; YU: Selva de Yungas.

Table 1. Species of Stevia studied. Political-administrative provinces. CAT: Catamarca; JUJ: Jujuy; SAL: Salta; LRI: La Rioja; TUC: Tucumán; CH: Chuquisaca; CO: Coquimbo; LP: La Paz; OR: Oruro; PO: Potosí; SC: Santa Cruz; TA: Tarija. Ecoregions. AA: High Andes; CS: Dry Chaco; MO: Mountain of Sierras and Bolsones (High Monte); PU: Puna; YU: Yungas.

\begin{tabular}{|c|c|c|c|c|c|c|}
\hline Especies & $\begin{array}{c}\text { Países y } \\
\text { subdivisiones } \\
\text { político- } \\
\text { administrativas }\end{array}$ & $\begin{array}{l}\text { Ecorregiones } \\
\text { de la } \\
\text { Argentina }\end{array}$ & $\begin{array}{c}\text { Altitud } \\
\text { (m s. n. m.) }\end{array}$ & Ambientes & Morfología & $\begin{array}{c}\text { Variedades } \\
\text { (de acuerdo } \\
\text { con Freire and } \\
\text { Ariza 2014) }\end{array}$ \\
\hline S. alpina & $\begin{array}{l}\text { Argentina: } \\
\text { CAT, JUJ, LRI, } \\
\text { SAL, TUC }\end{array}$ & $\mathrm{CS}, \mathrm{MO}, \mathrm{YU}$ & 1000-3100 & $\begin{array}{l}\text { Pastizales húmedos } \\
\text { de laderas, } \\
\text { matorrales, bosques } \\
\text { de aliso, selva, abras } \\
\text { y quebradas }\end{array}$ & $\begin{array}{l}\text { Subarbusto, hojas } \\
\text { pecioladas y ovadas, } \\
\text { papus formado por } \\
\text { escamas y 1-3 aristas }\end{array}$ & $\begin{array}{l}\text { var. alpina y } \\
\text { var. glutinosa }\end{array}$ \\
\hline S. breviaristata & $\begin{array}{l}\text { Argentina: } \\
\text { CAT, JUJ, LRI, } \\
\text { SAL, TUC. } \\
\text { Bolivia: SC, TA. }\end{array}$ & CS, YU & $450-3500$ & $\begin{array}{l}\text { Pastizales húmedos } \\
\text { de laderas, } \\
\text { montañas, serranías, } \\
\text { abras y valles secos }\end{array}$ & $\begin{array}{l}\text { Hierbas perennes } \\
\text { con hojas pecioladas } \\
\text { y ovadas, papus con } \\
\text { escamitas y } 1-2 \text { aristas }\end{array}$ & - \\
\hline S. minor & $\begin{array}{l}\text { Argentina: } \\
\text { CAT, JUJ, LRI, } \\
\text { MEN, SAL, } \\
\text { TUC. } \\
\text { Bolivia: CH, } \\
\text { CO, LP, OR, } \\
\text { PO. }\end{array}$ & AA, MO, PU & $3000-4500$ & $\begin{array}{l}\text { Habita en las } \\
\text { montañas, valles } \\
\text { secos y puna } \\
\text { húmeda }\end{array}$ & $\begin{array}{l}\text { Hierba perenne, hojas } \\
\text { sésiles y elípticas, } \\
\text { papus con escamitas y } \\
\text { 3-8 aristas (a veces } 15 \text { ) }\end{array}$ & - \\
\hline S. vaga & $\begin{array}{l}\text { Argentina: } \\
\text { CAT, JUJ, LRI, } \\
\text { SAL, SJU, TUC }\end{array}$ & MO, YU & $300-3100$ & $\begin{array}{l}\text { Abras, cerros, sierras } \\
\text { y montañas }\end{array}$ & $\begin{array}{l}\text { Subarbustos con } \\
\text { xilopodio, hojas } \\
\text { pecioladas o } \\
\text { pseudo-pecioladas, } \\
\text { lanceoladas, papus con } \\
\text { escamitas y 1-4 aristas }\end{array}$ & $\begin{array}{l}\text { var. effusa, var. } \\
\text { saltensis y var. } \\
\text { vaga }\end{array}$ \\
\hline S. yaconensis & $\begin{array}{l}\text { Argentina: } \\
\text { CAT, JUJ, SAL, } \\
\text { SJU, TUC. } \\
\text { Bolivia: CH, } \\
\text { CO, LP, SC, TA }\end{array}$ & $\begin{array}{l}\text { CS, MO, PU, } \\
\text { YU }\end{array}$ & 1000-3000 & $\begin{array}{l}\text { Pastizales serranos } \\
\text { de laderas húmedas, } \\
\text { prados serranos, } \\
\text { bosques de aliso, } \\
\text { quebradas, valles } \\
\text { secos y pajonales }\end{array}$ & $\begin{array}{l}\text { Hierbas perennes, } \\
\text { brevemente pecioladas } \\
\text { y ovado-lanceoladas, } \\
\text { papus con escamitas y } \\
1-3 \text { aristas }\end{array}$ & $\begin{array}{l}\text { var. } \\
\text { aristifera, var. } \\
\text { saltensis, var. } \\
\text { subeglandulosa } \\
\text { y var. } \\
\text { yaconensis }\end{array}$ \\
\hline
\end{tabular}

región, es necesario que dichas especies sean estudiadas con más profundidad. También se descartaron del análisis los especímenes de dudosa identificación, los duplicados y aquellos con información imprecisa sobre el lugar de recolección.

\section{Datos climáticos}

Los datos climáticos actuales y futuros fueron extraídos de la base de datos WorldClim (www.worldclim.org), generados a partir de la interpolación de bases de datos climáticos globales con datos desde el año 1950-2000 (Hijmans et al. 2005). Se utilizaron las 19 variables bioclimáticas disponibles en el modelo, tanto en condiciones actuales como futuras, con una resolución de 30 arcsec. El modelo aplicado para la proyección de estas coberturas fue MIROC5 por representar mejor el clima del NOA (Barros et al. 2005; Cuyckens et al. 2016). Se seleccionaron dos escenarios de concentración de gases de efecto invernadero para el año 2050, modelados por el IPCC en el Quinto Informe de Evaluación (AR5). En este trabajo, los escenarios seleccionados para analizar fueron rcp2.6 y rcp8.5, que representan dos situaciones extremas de emisión de gases contaminantes para el siglo XXI. El escenario rcp2.6 consiste en una situación en la que la emisión tiene un máximo para el año 2020 (van Vuuren et al. 2011), mientras que el escenario rcp8.5 proyecta una emisión continua a lo largo de todo el siglo (Riahi et al. 2011).

\section{Modelado de distribución potencial y distribución futura}

Las localidades puntuales fueron transformadas a información de mallas (Aagesen et al. 2009) por medio de herramientas GIS (QGIS y DIVA-GIS). Se 
exploró el tamaño apropiado de píxeles para la malla según el protocolo de Scheldeman y van Zonneveld (2010), y se decidió utilizar aquellas de $1 \mathrm{~km}^{2}$ (30 arcsec) dado que brindan mayor resolución. Se modeló la relación entre las variables climáticas y la distribución de las especies seleccionadas de Stevia utilizando un modelo de máxima entropía (Maxent v. 3.3.3k; Phillips et al. 2006; Phillips and Dudík 2008). El método de máxima entropía (Maxent) es muy utilizado porque es capaz de generar una mejor representación cuando sólo se utilizan puntos de presencia; su algoritmo matemático es conciso y su interpretación estadística, bien comprendida (Trethowan et al. 2001; Elith et al. 2006; Phillips et al. 2006; Kumar and Stohlgren 2009; Wolmarans et al. 2010; Merow et al. 2013). Asimismo, otros métodos de modelado se usan, por ejemplo, para análisis de riesgo territorial en especies invasoras (Heit et al. 2013). Aunque el empleo de diferentes técnicas de modelado puede generar resultados disímiles (e.g., Pearson et al. 2006; Fitzpatrick et al. 2013), se considera que Maxent es un método robusto y conservador para modelar la distribución de especies, en particular cuando se compara con métodos similares como GARP (Dudík et al. 2007; Qin et al. 2015).

Para circunscribir el área de estudio se generó una malla que contuviera las provincias de Catamarca, Jujuy, La Rioja, Salta y Tucumán, y se seleccionaron las variables climáticas más relevantes para esa región. En el caso de existir correlación entre dos o más variables (Pearson: $r>0.9$ ), para evitar la sobreexplicación de los factores abióticos que afectan la idoneidad de las especies en el área de estudio, se descartó el conjunto de variables que tuvieran el menor valor explicativo según las características fenológicas de las especies. Para ello se obtuvieron los datos climáticos de 3500 puntos aleatorios distribuidos en el área de estudio y se analizaron con el programa InfoStat (www.infostat.com.ar).

El programa Maxent se ejecutó con la siguiente configuración: número de repeticiones $=15$, puntos de fondo $=10000$, multiplicador de regularización $=1$, iteraciones $=500$. Se removieron las presencias duplicadas y las repeticiones se hicieron con validación cruzada ("crossvalidate"). Se utilizó un archivo de sesgo, generado a partir de un área de buffer de 1.5 grados alrededor de cada punto para cada especie. Se emplearon las características Linear, Quadratic, Product y Hinge, y el formato de salida elegido fue el logístico. Al utilizar sólo datos de presencia, la prevalencia no puede ser estimada de forma directa (Ward et al. 2009; Elith. et al. 2011). Por ello, se consideró una prevalencia de 0.5 , que es un valor empíricamente mejor calibrado para la salida logística de Maxent (Phillips and Dudík 2008; Elith. et al. 2011). Asimismo, se crearon las curvas de respuesta para las variables y se utilizó Jack-knife para medir la importancia explicativa de las variables. Se probaron diferentes valores para el multiplicador de regularización, buscando aquel que redujera los valores en las tasas de omisión ("omission rates") y que al mismo tiempo diera el mayor coeficiente de AUC (Radosavljevic and Anderson 2014). Al haber resultados similares para diferentes valores, se utilizó el menor (i.e., 1) para evitar la subestimación del modelo. De esta manera se obtuvieron 15 resultados diferentes para cada especie, generados a partir de un conjunto diferentes de datos para cada corrida. Al remover los registros duplicados por especie se evita la sobreestimación de las características climáticas de un mismo punto, dos veces. El área buffer garantiza que el programa no genere pseudo-ausencias en zonas en donde la planta podría distribuirse, pero no se la ha relevado (Mateo et al. 2010). Si bien el área definida fue aleatoria, Stevia tiene un gran poder dispersivo debido a la forma y la función del papus de la cipsela, por lo que se optó por una distancia de $~ 160$ $\mathrm{km}\left(1.5^{\circ}\right)$ alrededor de cada punto. Para el análisis de píxeles y la comparación entre los mapas de aptitud actual y futura se usaron los mapas promedio que genera Maxent sobre la base de cada réplica. El valor explicativo del grupo de variables fue evaluado por medio de los gráficos de área bajo la curva (AUC) que brinda Maxent. Los mapas fueron analizados a través de las herramientas QGIS (Quantum GIS Development Team 2017) y DIVA-GIS. La aptitud o idoneidad de hábitat toma valores entre 0 y 1 . Para su mejor interpretación visual se crearon cinco rangos de aptitud a partir de los valores obtenidos del mapa promedio para cada especie.

Para los modelos generados a partir de variables climáticas futuras, se realizó "clamping" con las variables. De esta forma se evita que algunas zonas del área de estudio en donde las variables climáticas puedan cambiar drásticamente en el futuro, tengan un impacto significativo en las proyecciones. Debido a que el número de puntos para algunas especies estudiadas no fue elevado $(n>15)$, se descartaron aquellas zonas que estén por fuera del rango representado por los puntos de entrenamiento. 


\section{Resultados}

El índice AUC varió en los modelos entre 0.7 y $0.95(?=0.84)$. Las variables de mayor contribución en los análisis fueron la precipitación en el período más lluvioso (BIO13), la temperatura promedio en el trimestre más seco (BIO09), el rango anual de temperatura (BIO07) y la estacionalidad en la temperatura (BIO04). A excepción del modelo

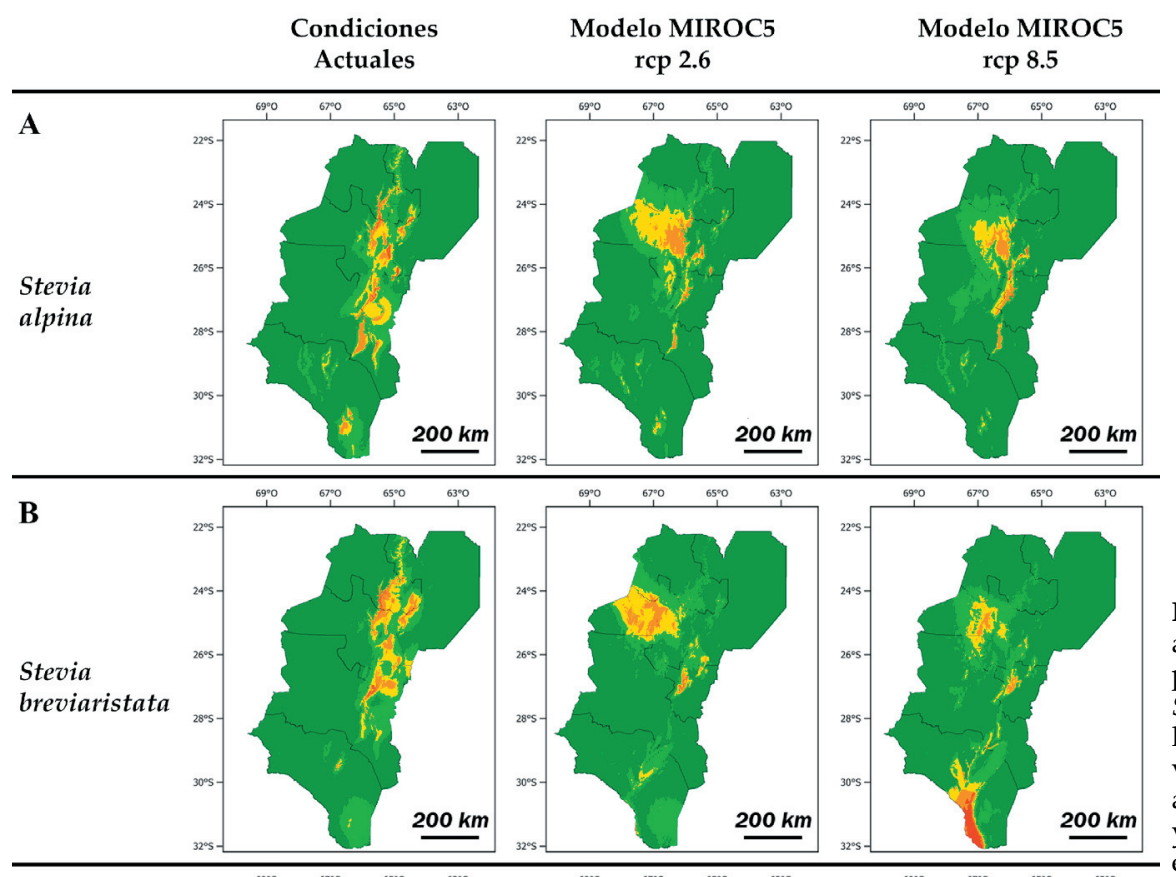

Figura 3. Mapas de aptitud de hábitat para las especies de Stevia. A la izquierda, los modelos con variables climáticas actuales; al centro y derecha, bajo un escenario de cambio

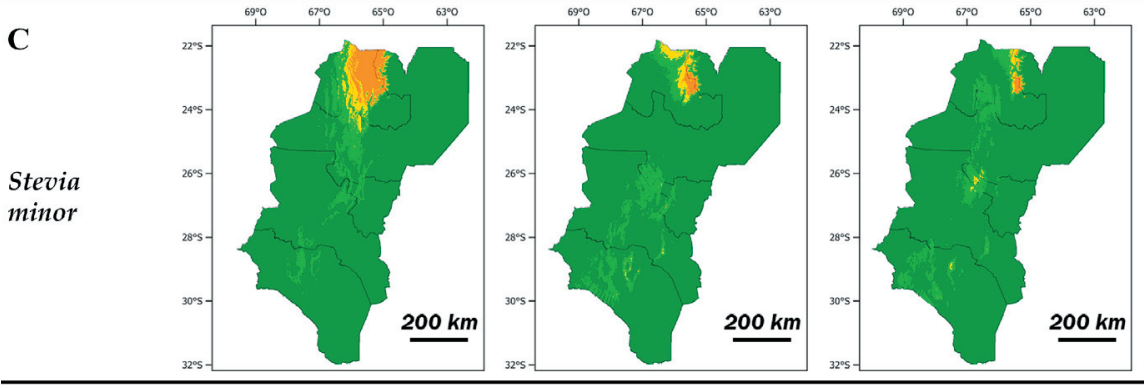
cimático. Modelos MIROC5 rcp2.6 y 8.5 , respectivamente, para el año 2050. A) S. alpina, B) $S$. breviaristata, C) $S$. minor, D) S. vaga, E) S. yaconensis (rangos de aptitud: verde oscuro $=0-0.3$; verde claro $=0.3-0.425$; amarillo $=0.425-0.65$; naranja $=0.65-0.825$;

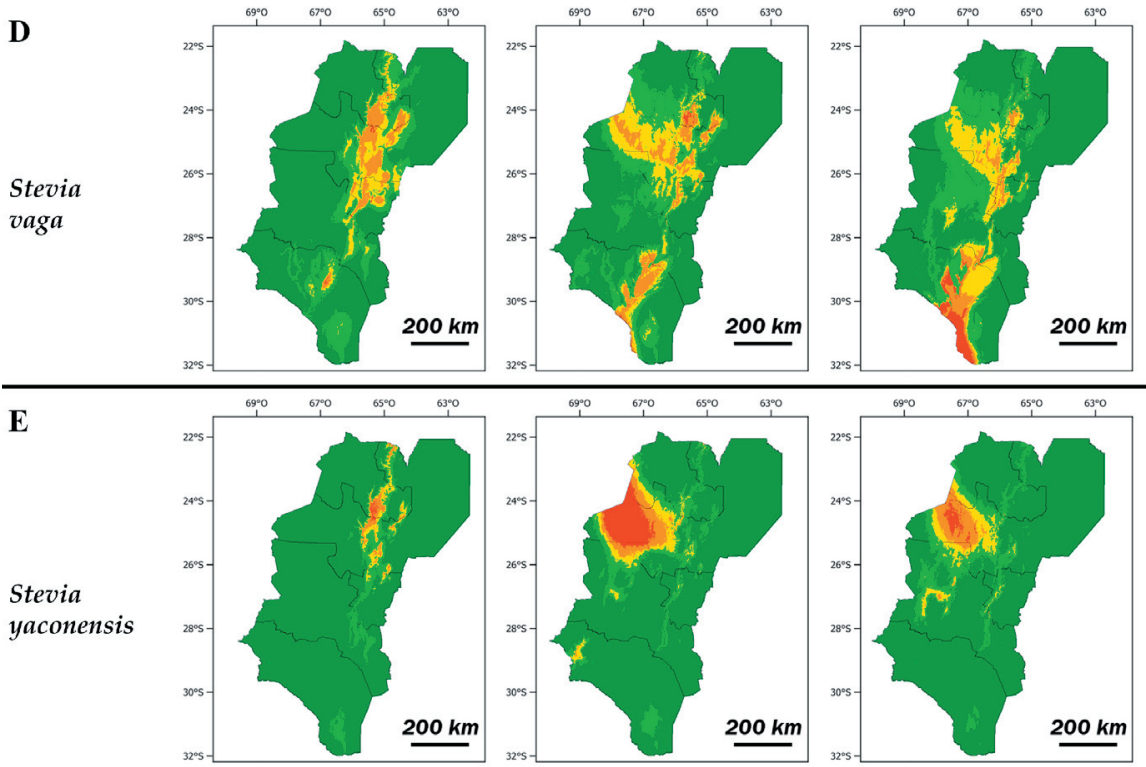
rojo=0.825-1).

Figure 3. Habitat suitability maps for Stevia species. On the left, generated models with current climatic variables; in the center and right, under a scenario of climate change. Models MIROC5 rcp2.6 and 8.5 , respectively, year 2050. A) S. alpina, B) S. breviaristata, C) $S$. minor, D) $S$. vaga, E) S. yaconensis (aptitude ranges: dark green $=0-0.3$; light green $=0.3-0.425$; yellow $=0.425-0.65$; orange $=0.65-0.825$; red $=0.825-1$ ). 
de S. minor donde la variable determinante fue la temperatura promedio en el trimestre más seco, la variable de mayor aporte para el resto de los modelos fue la precipitación en el período más lluvioso.

Las tendencias observadas en ambos escenarios climáticos del modelo MIROC5 fueron similares. Cuando se los compara, el escenario rcp8.5 fue más severo en las reducciones, lo que suele reflejarse como un mayor porcentaje en la reducción de píxeles aptos de la especie cuando se lo contrastó con el modelo climático actual (Figura 3).

Las especies S. alpina, S. breviaristata, S. vaga y S. yaconensis bajo el modelo rcp8.5 (Figura 3A, B, D, E), se desplazaron hacia el oeste, hasta los Andes occidentales. Esto resultó en una reducción distribucional en la ecorregión de la Selva de Yungas y una expansión hacia las otras ecorregiones, en particular Puna, Altos Andes y los Montes de Sierras y Bolsones.

El número total de píxeles de aptitud (valor $>0.3$ ) aumentó para las especies $S$. vaga y $S$. yaconensis (Figura 3D, E). Además, la aptitud general de ambas especies se vio favorecida, especialmente en el escenario rcp2.6, con aumento de pixeles con valor mayor a 0.65 en un $32 \%$ para la primera y tres veces su área para $S$. yaconensis. Por el contrario, las especies S. alpina, S. breviaristata y S. minor (Figura 3A, $B, C)$ redujeron el número total de pixeles de aptitud y también los rangos de mayor aptitud. El caso más drástico se observó en $S$. minor para el escenario climático rcp8.5. Esta especie experimentó una reducción de hasta $89 \%$ para pixeles con valor mayor a 0.65 .

Las zonas de mayor aptitud se trasladaron en gran medida hacia el sur del área de estudio en $S$. breviaristata y $S$. vaga, bajo el escenario rcp8.5, con valores de pixeles mayores a 0.65 en la provincia de La Rioja (Figura 3B, D). Stevia breviaristata conservó un nodo de idoneidad de hábitat en el extremo oeste de Tucumán cuando se compara la proyección con el mapa para condiciones actuales. Mientras tanto, $S$. vaga desplazó su aptitud hacia el sur del área de estudio bajo el escenario rcp8 de cambio climático (Figura 3).

\section{DiscusióN}

El cambio climático afecta de manera profunda la distribución de las especies y los ecosistemas (Midgley et al. 2002; Higgins 2007). Según nuestros modelos, todas las especies de Stevia modificarían su rango de aptitud de hábitat para el año 2050.

Los resultados observados en 4 de las 5 especies aquí analizadas concuerdan con las tendencias observadas en otras especies endémicas del área: se desplazarían sobre todo en dirección este-oeste (Parmesan 2006), y no en dirección sur-norte (Godoy-Bürki 2016). La migración se observa mayormente en aquellas especies que amplían el área de idoneidad de hábitat. Este fenómeno se conoce como un desplazamiento en altura (Feeley and Silman 2010), en el que las especies migran de zonas más bajas y cálidas hacia las zonas de mayor altitud, frescas y húmedas. Sin embargo, se debe tener en cuenta que los modelos no predicen las capacidades adaptativas, de dispersión o la modificación del suelo, por lo que no garantizan que las especies vayan a lograr alcanzar estos sitios (Godoy-Bürki 2016). Para aquellas especies que amplían su área de aptitud, este fenómeno podría explicarse por el hecho de que las poblaciones actualmente viven en climas que son más frescos que su temperatura fisiológica óptima. De esta forma, el calentamiento podría, incluso, mejorar su condición física (Deutsch et al. 2008). Se necesitarían nuevos análisis con metodologías diferentes a Maxent para evaluar la confianza de las tendencias de cambio de nicho climático reflejadas en este trabajo.

En particular, las especies que se distribuyen en la ecorregión de las Yungas (S. alpina, $S$. breviaristata, S. vaga y S. yaconensis), tienden a modificar suárea de aptitud conforme aumenta la rigurosidad del modelo climático. Los cambios en las áreas con condiciones climáticas favorables son considerables en las regiones con clima templado a semiárido. El norte de Salta y sur de Jujuy, donde se encuentran las Selvas Montanas y Pedemontanas (Mendoza and González 2011), se verían principalmente afectadas, al igual que la zona de transición con el Chaco Seco en la región norte de Tucumán. Esto podría relacionarse con la predicción en la disminución de las precipitaciones, conforme estas áreas se caracterizan por poseer un clima templado de verano fresco a semiárido de estepa (Mendoza and González 2011).

Por otro lado, S. minor fue registrada en ambientes xerófitos de alta montaña con precipitaciones menores a $250 \mathrm{~mm}$ anuales, como ya se mencionara antes. Bajo el escenario rcp8.5, esta especie sufriría una reducción drástica (mayor a $80 \%$ ) para valores de aptitud superiores a 0.65 , y quedaría restringida a una 
pequeña área del centro-norte de Jujuy y Salta (Figura 3). No obstante, a pesar de registrar una reducción en el área con condiciones climáticas favorables, el impacto del cambio climático sobre las ecorregiones áridas sería menor que en las zonas más húmedas (GodoyBürki 2016). El desplazamiento proyectado para estas especies de Stevia en el NOA, aun cuando en algunas de ellas se produzcan reducciones en el rango total de idoneidad de hábitat, sugiere que esta región podría funcionar como refugio frente a los cambios climáticos en el futuro dada la amplitud de tolerancia climática de las especies estudiadas y la heterogeneidad ambiental de la región del NOA. Trabajos previos mostraron que la región estudiada habría funcionado como área de refugio de especies durante las glaciaciones del Cuaternario (Sandel et al. 2011; Corlett and Westcott 2013).

La base climática de Worldclim (Hijmans et al. 2005) tiende a contener errores predictivos en áreas con menor disponibilidad de estaciones meteorológicas en escalas locales, lo cual fue analizado para temperatura y precipitación media anual en pendientes orientales de los Andes tropicales (Deblauwe et al. 2016) y en la Puna seca (Bennett et al. 2016). Sin embargo, modelos realizados sobre la base de datos climáticos permitieron registrar nuevas poblaciones de especies poco colectadas en áreas montañosas de los Andes, tanto en plantas como en animales (e.g., Botero-Delgadillo et al. 2013; Särkinen et al. 2013; Armesto et al. 2015). La influencia del cambio climático en las especies del NOA y el Altiplano aún es muy poco conocida.

El género Stevia posee una elevada riqueza de especies que habitan en una gran diversidad de ambientes. Por ello, los resultados aquí obtenidos son limitados y no deberían generalizarse al resto de las especies que habitan en el noroeste argentino, sino que deberían servir de punto de partida para futuros estudios en este taxón. Estudios posteriores que consideren el rango total de distribución de las especies aquí modeladas e incluyan un mayor número de especies de Stevia podrán comprobar si existen tendenciaso patrones geográficos generales. Además, sería interesante incluir otras variables climáticas y topográficas que permitan reconocer mejor el nicho de hábitat de estas especies.

Finalmente, y en el marco de la revisión del género Stevia para la Argentina que está llevando a cabo el primer autor, las hipótesis geográficas obtenidas de la distribución potencial actual de las cinco especies servirán para explorar nuevas localidades de probables ocurrencias, así como también monitorear y evaluar las poblaciones conocidas.

Agradecimientos. Agradecemos especialmente a S. Montemayor, F. Biganzoli, P. Pliscoff y C. Abarca por la lectura crítica del manuscrito o consejos en el manejo de software, y a los revisores anónimos por los comentarios y recomendaciones para mejorar el trabajo. Se agradece también a L. J. Novara por las fotografías de las especies estudiadas en su ambiente. Asimismo, a los curadores de los herbarios BAF (G. Giberti), CORD (G. Barboza), CTES (M. Dematteis), LIL (N. Muruaga), LP (L. Iharlegui) y SI (N. Deginani) por el préstamo de especímenes y su buena disposición y ayuda. Se contó con financiamientos de la ANPCYT, el CONICET y la UNLP. Se agradece al Consejo Interuniversitario Nacional (CIN) dado que la investigación inicial se realizó en el marco de una beca otorgada por dicho consejo al primer autor.

\section{REFERENCIAS}

Aagesen L., C. A. Szumikb, F. O. Zuloaga, and O. Morrone. 2009. Quantitative biogeography in the South America highlands - Recognizing the Altoandina, Puna and Prepuna through the study of Poaceae. Cladistics 24:1-16.

Aagesen, L., M. J. Bena, S. Nomdedeu, A. Panizza, R. P. López, and F. O. Zuloaga. 2012. Areas of endemism in the southern central Andes. Darwiniana 50:218-251.

Armesto, L. O., E. Quilarque, and F. J. M. Rojas-Runjaic. 2015. New locality records and geographic distribution map of Dendropsophus meridensis (Rivero, 1961) (Anura: Hylidae) in the Andes of Venezuela. Check List 11(1):1-5.

Barros, V., C. Vera, E. Agosta, D. Araneo, I. Camilloni, A. Carril, M. Doyle, O. Frumento, M. Nuñez, M. Ortiz De Zárate, O. Penalba, M. Rusticucci, C. Saulo, and S. Solman. 2015. Cambio Climático en Argentina; tendencias y proyecciones. $3^{\circ}$ Comunicación Nacional de la República Argentina a la Convención Marco de las Naciones Unidas sobre Cambio Climático. Centro de Investigaciones del Mar y la Atmósfera. Anexo II.

Bennett, M., M. New, J. Marino, and C. Sillero. 2016. Climate complexity in the Central Andes: A study case on empirically-based local variations in the Dry Puna. Journal of Arid Environments 128:40-49.

Beniston, M. 1997. Variations of Snow Depth and Duration in the Swiss Alps over the last 50 Years: Links to Changes in Large-scale Climatic Forcings, Climatic Change 36:281-300.

Biganzoli, F., C. Larsen, and A. G. Rolhauser. 2013. Range expansion and potential distribution of the invasive grass Bromus tectorum in southern South America on the base of herbarium records. Journal of Arid Environments 97: 
230-236.

Botero-Delgadillo, E., C. A. Páez, and J. Sanabria-Mejía. 2012. Discovery of two new localities for Todd's parakeet Pyrrhura picta caeruleiceps using distribution models: Enhancing knowledge of a little known neotropical bird. Ardeola 59:237-252.

Brown, D. A, and S. Pacheco. 2006. Propuesta de actualización del mapa ecorregional de la Argentina. Pp. 28-31 en D. A. Brown, U. Martínez Ortiz, M. Acerbi and J. Corcuera (eds.). La Situación Ambiental Argentina 2005. Fundación Vida Silvestre Argentina, Buenos Aires, Argentina.

Bush, M. B. 2002. Distributional change and conservation on the Andean flank: a palaeoecological perspective. Global Ecology and Biogeography 11:463-473.

Cabrera, A. L. 1976. Regiones fitogeográficas argentinas. Pp. 1-85 en W. F. Kugler (ed.). Enciclopedia argentina de agricultura y jardinería, Tomo 2(1). Acme, Buenos Aires, Argentina.

Cabrera, A. L. 1978. Compositae. Pp. 1-726 en A. L. Cabrera (ed.). Flora de la Provincia de Jujuy. Colección Científica del Instituto Nacional de Tecnología Agropecuaria 13(10). INTA, Buenos Aires, Argentina.

Cabrera, A. L., and S. E. Freire. 1997. Asteraceae, Tribu II. Eupatorieae (excepto Mikania). Pp. 1-54, 76-104 en A. T. Hunziker (ed.). Flora Fanerogámica Argentina 47. PROFLORA CONICET, Buenos Aires, Argentina.

Carrilla, J., H. R. Grau, L. Paolini, and M. Morales. 2013. Lake fluctuations, Plant productivity, and Long-Term Variability in High-Elevation Tropical Andean Ecosystems. Arctic, Antarctic, and Alpine Research 45(2):179-189.

Coitiño, H. I., F. Montenegro, A. Fallabrino, E. M. González, and D. Hernández. 2013. Distribución actual y potencial de Cabassous tatouay y Tamandua tetradactyla en el límite sur de su distribución: implicancias para su conservación en Uruguay. Edentata 14:23-34.

Corlett, R. T., and D. A. Westcott. 2013. Will plant movements keep up with climate change? Trends in Ecology and Evolution 28(8):482-8.

Cuesta-Camacho, F., M. Peralvo, and A. Ganzenmüller. 2008. Posibles efectos del calentamiento global sobre el nicho climático de algunas especies en los Andes Tropicales. Páramo y Cambio Climático 23:15-38.

Cuyckens, G. A. E., D. A. Christie, A. I. Domic, L. R. Malizia, and D. Renison. 2016. Climate change and the distribution and conservation of the world's highest elevation woodlands in the South American Altiplano. Global and Planetary Change 137:79-87.

Deblauwe, V., V. Droissart, R. Bose, and T. L. P. Couvreur. 2016. Remotely sensed temperature and precipitation data improve species distribution modelling in the tropics. Global Ecology and Biogeography 25(4):443-454.

Deutsch, C. A., J. J. Tewksbury, R. B. Huey, K. S. Sheldon, C. K. Ghalambor, et al. 2008. Impacts of climate warming on terrestrial ectotherms across latitude. Proceedings of the National Academy of Sciences of the United States of America 105:6668-6672.

Dudík, M., S. J. Phillips, and R. E. Schapire. 2007. Maximum entropy density estimation with generalized regularization and an application to species distribution modeling. Journal of Machine Learning Research 8:1217-1260.

Elith, J., C. H. Graham, R. P. Anderson, M. Dudík, S. Ferrier, A. Guisan, R. J. Hijmans, F. Huettmann, J. R. Leathwick, A. Lehmann, J. Li, L. G. Lohmann, B. A. Loiselle, G. Manion, C. Moritz, M. Nakamura, Y. Nakazawa, J. M. M. Overton, A. Townsend Peterson, S. J. Phillips, K. Richardson, R. Scachetti-Pereira, R. E. Schapire, J. Soberón, S. Williams, M. S. Wisz, and N. E. Zimmermann. 2006. Novel methods improve prediction of species' distributions from occurrence data. Ecography 29:129-151.

Elith, J., S. J. Phillips, T. Hastie, M. Dudík, Y. E. Chee, and C. J. Yates. 2011. A statistical explanation of MaxEnt for ecologists. Diversity and Distributions 17:43-57.

Feeley, K. J, and M. R. Silman. 2010. Land-use and climate change effects on population size and extinction risk of Andean plants. Global Change Biology 16:1-8.

Ferrier, S., and A. Guisan. 2006. Spatial modelling of biodiversity at the community level. Journal of Applied Ecology 43:393-404.

Fitzpatrick, M. C., N. J. Gotelli, and A. M. Ellison. 2013. MaxEnt versus MaxLike: empirical comparisons with ant species distributions. Ecosphere 4(5):1-15.

Foster, P. 2001. The potential negative impacts of global change on tropical montane cloud forests. Earth-Science Reviews 55:73-106.

Freire, S. E. 2008. Stevia. Pp. 1509-1517 en F. Zuloaga, O. Morrone and M. J. Belgrano (eds.). Catálogo de las Plantas Vasculares del Cono Sur (Argentina, Sur de Brasil, Chile, Paraguay y Uruguay). Monographs in Systematic Botany from the Missouri Botanical Garden 107(2). Estudio Sigma S.R.L., Buenos Aires, Argentina.

Freire, S. E., G. Sancho, E. Urtubey, N. D. Bayón, L. Katinas, D. A. Giuliano, D. G. Gutiérrez, A. A. Sáenz, L. Iharlegui, C. Monti, and G. Delucchi. 2005. Catalogue of Asteraceae of Chacoan plain, Argentina. Compositae Newsletter 43: $1-126$.

Freire, S. E., and A. M. Molina. 2009. Flora Chaqueña-Argentina: Formosa, Chaco y Santiago del Estero. Familia Asteraceae. Ediciones INTA, Buenos Aires.

Freire, S. E., N. D. Bayón, C. Monti, D. A. Giuliano, L. Ariza Espinar, A. A. Sáenz, M. V. Perea, and G. Delucchi. 2011. Sinopsis de las Asteraceae de la Provincia de Catamarca. Editorial Científica de la Universidad Nacional de Catamarca, Catamarca, Argentina. Pp. 235.

Freire, S. E., and L. Ariza Espinar. 2014. Stevia. Pp. 409-435 en F. O. Zuloaga, M. J. Belgrano and A. M. Anton (eds.). Flora Vascular de la República Argentina 7(1). Estudio Sigma SRL, Buenos Aires.

Godoy-Bürki, A. C. 2015. Diversidad de plantas vasculares en zonas áridas del Noroeste de Argentina (NOA): Patrones de Distribución, Prioridades de Conservación y Cambio climático. Tesis Doctoral. Doctor en Biología. Facultad de Ciencias Naturales y Museo, Universidad Nacional de La Plata, Argentina. Pp. 147.

Godoy-Bürki, A. C. 2016. Efectos del cambio climático sobre especies de plantas vasculares del sur de los Andes 
Centrales: un estudio en el noroeste de Argentina (NOA). Ecología Austral 26:83-94.

Godoy-Bürki, A. C., F. Biganzoli, J. M. Sajama, P. Ortega-Baes, and L. Aagesen. 2017. Tropical high Andean drylands: species diversity and its environmental determinants in the Central Andes. Biodiversity and Conservation. doi: 10.1007/s10531-017-1311-2.

González, J. A. 2005. Los ambientes naturales en áreas montañosas del noroeste argentino, su interrelación con países limítrofes y su necesidad de protección, recuperación y conservación. Serie Conservación de la Naturaleza N ${ }^{\circ} 15$. Pp. 28

González, J. A. 2009. Climatic change and other anthropogenic activities are affecting environmental services on the Argentina Northwest (ANW). Environmental Earth Sciences 6:1-2.

Grau, R. H., I. N. Gasparri, and M. T. Aide. 2005. Agriculture expansion and deforestation in seasonally dry forests of north-west Argentina. Environmental Conservation 32:140-148.

Guisan, A., and W. Thuiller. 2005. Predicting species distribution: offering more than simple habitat models. Ecology Letters 8:993-1009.

Gutiérrez, D. G., M. Muñoz-Schick, M. Grossi, J. F. Rodriguez-Cravero, V. Morales, and A. Moreira-Muñoz. 2016. The genus Stevia (Eupatorieae, Asteraceae) in Chile: a taxonomical and morphologic analysis. Phytotaxa 282:1-18.

Grossi, M. A., D. G. Gutiérrez, and G. Delucchi. 2012. Una mirada sobre el estado actual de la conservación de la flora argentina. Conservación Vegetal 16:15-17.

Heit, G., W. Sione, P. Aceñolaza, L. Zamboni, P. Blanco, P. Horak, and P. Cortese. 2013. Modelo de distribución potencial de Lobesnia botrana (Lepidoptera: Tortricidae). Una herramienta de planificación para su detección temprana a nivel regional. Geofocus: International Review of Geographical Information Science and Technology 13(2):179-194.

Hijmans, R. J., S. E. Cameron, J. L. Parra, P. G. Jones, and A. Jarvis. 2005. Very high resolution interpolated climate surfaces for global land areas. International Journal of Climatology 25:1965-1978.

Higgins, P. A. T. 2007. Biodiversity loss under existing land use and climate change: an illustration using northern South America. Global Ecology and Biogeography 16:197-204.

Hind, D. J. N., and H. E. Robinson. 2007. Tribe Eupatorieae. Pp. 510-574 en Kubitzki, K. (ed.). The Families and Genera of Vascular Plants vol. VIII. Springer-Verlag. Berlin, Heidelberg, Alemania.

Hutchinson, G. E. 1957. Concluding remarks, Cold Spring Harbor Symposium. Quantitative Biology 22:415-427.

IPCC. 2007. Chapter 10: Global Climate Projections. Pp. 747-844 in S. Solomon, D. Qin, M. Manning, Z. Chen, M. Marquis, K. B. Averyt, M. Tignor and H. L. Miller (eds.). Climatic Change 2007: The Physical Science Basis. Contribution of Working Group I to Fourth Assessment Report of The Intergovernmental Panel on Climate Change (IPCC). Cambridge University Press, Cambridge, United Kingdom and New York, NY, USA.

Jarvis, A., K. Williams, D. Williams, L. Guarino, P. J. Caballero, and G. Mottram. 2005. Use of GIS for optimizing a collecting mission for a rare wild pepper (Capsicum exuosum Sendtn.) in Paraguay. Genetic Resources and Crop Evolution 52:671-682.

Katinas L., D. G. Gutiérrez, M. A. Grossi, and J. V. Crisci. 2007. Panorama de la familia Asteraceae (= Compositae) en la República Argentina. Boletín de la Sociedad Argentina de Botánica 42:113-129.

Köppen, W. 1923. Die Klimate der Erde. Grundiriss deer Klimakunde. X+, Berlín und Leipzig. Pp. 369.

Kumar, S., and T. J. Stohlgren. 2009. Maxent modeling for predicting suitable habitat for threatened and endangered tree Canacomyrica monticola in New Caledonia. Journal of Ecology and The Natural Environment 1:94-98.

Malcolm, J. R., C. Liu, R. P. Neilson, L. Hansen, and L. Hannah. 2006. Global warming and extinctions of endemic species from biodiversity hotspots. Conservation Biology 20:538-548.

Maldonado, C., C. I. Molina, A. Zizka, C. Persson, C. M. Taylor, J. Albán, E. Chiquillo, N. Rønsted, and A. Antonelli. 2015. Estimating species diversity and distribution in the era of Big Data: To what extent can we trust public databases? Global Ecology and Biogeography 24:973-984.

Marengo, J. A., J. D. Pabón, A. Díaz, G. Rosas, G. Ávalos, et al. 2011. Climate change: evidence and future scenarios for the Andean region. Pages 110-127 in S. K. Herzog, R. Martínez, P. M. Jorgensen and H. Tiessen (eds.). Climate Change and biodiversity in the tropical Andes. Inter-American Institute of Global Change Research and Scientific Committee on Problems of the Environment (SCOPE).

Mateo, R. G., T. B. Croat, A. M. Felicísimo, and J. Muñóz. 2010. Profile or group discriminative techniques? Generating reliable species distribution models using pseudo-absences and target-group absences from natural history collections. Diversity and Distributions 16:84-94.

Mendoza, E. A., and J. A. González. 2011. Las ecorregiones del Noroeste Argentino basadas en la clasificación climática de Köppen. Serie Conservación de la Naturaleza 19:3-41.

Merow, C., M. J. Smith, and J. A. Silander. 2013. A practical guide to MaxEnt for modeling species' distributions: what it does, and why inputs and settings matter. Ecography 36:1058-1069.

Midgley, G. F., L. Hannah, D. Millar, M. C. Rutherford, and L. W. Powrie. 2002. Assessing the vulnerability of species richness to anthropogenic climate change in a biodiversity hotspot. Global Ecology and Biogeography 11:445-451.

Minetti, J. L. 2005. El Clima del Noroeste Argentino. Magna, San Miguel de Tucumán. Pp. 449.

Minetti, J. L., and J. A. González. 2006. El cambio climático en la provincia de Tucumán: sus impactos. Serie Conservación de la Naturaleza 17:1-32.

Minvielle, M., and R. D. Garreaud. 2011. Projecting rainfall changes over the South American Altiplano. Journal of Climate 24:4577-4583.

Morales, M. S., J. Carilla, H. R. Grau, and R. Villalba. 2015. Multi-century lakes area changes in the Southern Altiplano: a tree-ring-based reconstruction. Climate of the Past 11:1139-1152.

Moritz, C., J. L. Patton, C. J. Conroy, J. L. Parra, G. C. White, and S. R. Beissinger. 2008. Impact of a Century of Climate 
Change on Small-Mammal Communities in Yosemite National Park, USA. Science 322:261-264.

Núñez, M. N., S. A. Solman, and M. F. Cabré. 2009. Regional climate change experiments over southern South America. II: Climate change scenarios in the late twenty-first century. Climate Dynamics 32:1081-1095.

Parmesan, C. 2006. Ecological and Evolutionary Responses to Recent Climate Change. Annual Review of Ecology, Evolution and Systematics 37:637-669.

Peterson, A. T., M. A. Ortega-Huerta, J. Bartley, V. Sánchez-Cordero, J. Soberon, R. H. Buddemeier, and D. R. B. Stockwell. 2002. Future projections for Mexican faunas under global climate change scenarios. Nature 416:626-269.

Pimm, S. L., G. J. Russell, J. L. Gittleman, and T. M. Brooks. 1995. The future of biodiversity. Science 269:347-350.

Pliscoff, P., and T. Fuentes-Castillo. 2011. Modelación de la distribución de especies y ecosistemas en el tiempo y en el espacio: una revisión de las nuevas herramientas y enfoques disponibles. Revista de Geografía Norte Grande 48: 61-79.

Phillips, S. J., R. P. Anderson, and R. E. Schapire. 2006. Maximum entropy modeling of species geographic distributions. Ecological Modelling 190:231-259.

Phillips, S. J., and M. Dudík. 2008. Modeling of species distributions with Maxent: new extensions and a comprehensive evaluation. Ecography 31:161-175.

Quantum GIS Development Team. 2017. Quantum GIS Geographic Information System. Open Source Geospatial Foundation Project. URL: qgis.osgeo.org.

Qin, Z., J. Zhang, A. Ditommaso, R. Wang, and R. Wu. 2014. Predicting invasions of Wedelia trilobata (L.) Hitchc. with Maxent and GARP models. Journal of Plant Research 128:763-775.

Radosavljevic, A., and R. P. Anderson. 2014. Making better MAXENT models of species distributions: complexity, overfitting and evaluation. Journal of Biogeography 41:629-643.

Riahi, K., S. Rao, V. Krey, C. Cho, V. Chirkov, G. Fischer, G. Kindermann, N. Nakicenovic, and P. Rafaj. 2011. RCP 8.5-A scenario of comparatively high greenhouse gas emissions. Climatic Change 109:33-57.

Sandel, B., L. Arge, B. Dalsgaard, R. G. Davies, K. J. Gaston, W. J. Sutherland, and J. C. Svenning. 2011. The influence of Late Quaternary climate-change velocity on species endemism. Science 334:660-664.

Särkinen, T., P- Gonzáles, and S. Knapp. 2013. Distribution models and species discovery: the story of a new Solanum species from the Peruvian Andes. Phytokeys 31:1-20.

Spencer, C., K. Yamamoto, J. Fang, H. Constable, M. Koo, and J. Wieczorek. 2006. Georeferencing for dummies. Traducido por S. Celis, G. Iglesias, and F. Uribe. Berkeley: University of California, California. USA. URL: www.herpnet.org/ herpnet/documents/georeffordummy.xls.

Scheldeman, X., L. Willemen, G. Coppens D'eeckenbrugge, E. Romejin-Peeters, M. T. Restrepo, J. Romero Motoche, D. Jiménez, M. Lobo, C. I. Medina, C. Reyes, D. Rodríguez, J. A. Ocampo, P. Van Damme, and P. Goetgebeur. 2006. Distribution, diversity and environmental adaptation of highland papayas (Vasconcellea spp.) in tropical and subtropical America. Biodiversity and Conservation 16:1867-1884.

Scheldeman X., and M. van Zonneveld. 2010. Manual de Capacitación en Análisis Espacial de Diversidad y Distribución de Plantas. Biodiversity International, Roma, Italia. Pp. 186.

Szumik, C., L. Aagesen, D. Casagranda, V. Arzamendia, D. Baldo, L. E. Claps, F. Cuezzo, J. M. Díaz Gómez, A. Di Giacomo, A. Giraudo, P. Goloboff, C. Gramajo, C. Kopuchian, S. Kretzschmar, M. Lizarralde, A. Molina, M. Mollerach, F. Navarro, S. Nomdedeu, A. Panizza, V. V. Pereyra, M. Sandoval, G. Scrocchi, and F. O. Zuloaga. 2012. Detecting areas of endemism with a taxonomically diverse data set: plants, mammals, reptiles, amphibians, birds, and insects from Argentina. Cladistics 28:317-329.

Thomas, C. D., A. Cameron, R. E. Green, M. Bakkenes, L. J. Beaumont, Y. C. Collingham, B. F. N. Erasmus, M. Ferreira De Siqueira, A. Grainger, L. Hannah, L. Hughes, B. Huntley, A. S. Van Jaarsveld, G. F. Midgley, L. Miles, M. A. Ortega-Huerta, A. Townsend Peterson, A. L. Phillips, and S. E. Williams. 2004. Extinction risk from climate change. Nature 427:145-148.

Trethowan, P., M. P. Robertson, and A. Mcconnachie. 2011. Ecological niche modeling of an invasive alien plant and its potential biological control agents. South African Journal of Botany 77:137-146.

Urrutia, R., and M. Vuille. 2009. Climate change projections for the tropical Andes using a regional climate model: Temperature and precipitation simulations for the end of the 21st century. Journal of Geophysical Research: Atmospheres 114:1-15.

Van Vuuren, D. P., E. Stehfest, M. G. J. den Elzen, T. Kram, J. van Vliet, S. Deetman, M. Isaac, K. Klein Goldewijk, A. Hof, A. Mendoza Beltrán, R. Oosternrijk, and B. van Ruijven. 2011. RCP2.6: exploring the possibility to keep global mean temperature increase below $2^{\circ} \mathrm{C}$. Climatic Change 109:95-116.

Vilatersana, R., M. Sanz, A. Gallan, and E. Castells. 2016. The invasion of Senecio pterophorus across continents: multiple, independent introductions, admixture and hybridization. Biological Invasions 18(7):2045-2065.

Vuille, M., B. Francou, P. Wagnon, I. Juen, G. Kaser, B. G. Mark, and R. S. Bradley. 2008. Climate change and tropical Andean glaciers: Past, present and future. Earth-Science Reviews 89:79-96.

Wolmarans, R., M. P. Robertson, and B. J. Van Rensburg. 2010. Predicting invasive alien plant distributions: how geographical bias in occurrence records influences model performance. Journal of Biogeography 37:1797-1810.

Young, K. E., L. B. Abbott, C. A. Caldwell, and T. S. Schrader. 2013. Estimating suitable environments for invasive plant species across large landscapes: A remote sensing strategy using Landsat 7 ETM+. International Journal of Biodiversity and Conservation 5:122-134. 